\title{
Neutrino Oscillations and Decoherence in Short-GRB Progenitors
}

\author{
A. V. Penacchioni ${ }^{1}$ (D) and O. Civitarese ${ }^{2}$ \\ ${ }^{1}$ IFLP (CONICET), La Plata, Argentina; ana.penacchioni@ fisica.unlp.edu.ar \\ 2 Department of Physics, University of La Plata (UNLP), 49 y 115 cc. 67, 1900 La Plata, Argentina \\ Received 2018 November 13; revised 2019 January 10; accepted 2019 January 13; published 2019 February 12
}

\begin{abstract}
Neutrinos are produced in cosmic accelerators, like active galactic nuclei, blazars, supernova remnants and gammaray bursts (GRBs). On their way to the Earth they experience flavor oscillations. The interactions of the neutrinos coming from the source with other particles, e.g., intergalactic primordial neutrinos or heavy-mass right-handed neutrinos, on their way to the detector may transform the original wavepacket in pointer states. This phenomenon, known as decoherence, becomes important in the reconstruction of processes at the source. In this work, we study neutrino emission in short GRBs by adopting the Fireshell Model. We consider $e^{-} e^{+}$-pair annihilation as the main channel for neutrino production. We compare the properties of the neutrino flux with the characteristic photon signal produced once the transparency condition is reached. We study the effects of flavor oscillations and decoherence as neutrinos travel from the region near the black hole event horizon outward. We consider the source to be in thermal equilibrium and calculate energy distribution functions for electrons and neutrinos. To compute the effects of decoherence we use a Gaussian model. In this scenario, the emitted electron-neutrinos transform into pointer states consisting of $67.8 \%$ electron-neutrinos and $32.2 \%$ as a combination of mu and tau neutrinos. We found that decoherence plays an important role in the evolution of the neutrino wavepacket, leading to the detected pointer states on Earth.
\end{abstract}

Key words: astroparticle physics - black hole physics - gamma-ray burst: general - neutrinos

\section{Introduction}

Short gamma-ray bursts (S-GRBs) are intense flashes of gamma-rays that last less than $2 \mathrm{~s}$ in the observer frame. It is widely accepted that S-GRBs originate from the merging of two compact objects, such as a neutron star (NS) and a black hole $(\mathrm{BH})$, or two neutron stars (NS-NS). During the merging phase, angular momentum and energy losses are manifested as gravitational wave emission and electromagnetic radiation. In both cases, the remnant is a $\mathrm{BH}$ of a few solar masses. There are different models that try to explain the observed emission of S-GRBs; among others, the Fireball Model (Piran 1999) and the Fireshell Model (Bianco et al. 2008a, 2008b; Bianco \& Ruffini 2008; Enderli et al. 2014).

The Fireball Model states that in the case of the NS-NS system an accretion disk is formed around the newly born $\mathrm{BH}$ (Berger 2014). In the NS-BH case, the same can occur if the NS is tidally disrupted outside the BH's event horizon. The rapidly rotating $\mathrm{BH}$ bends the magnetic field lines forming a double jet perpendicular to the accretion-disk plane. A fraction of the electromagnetic radiation escapes in the form of gammarays, while another fraction goes into neutrino-antineutrino emission (Narayan et al. 1992).

In the Fireshell Model scenario, the NS-NS merging leads to a massive NS that exceeds its critical mass and gravitationally collapses to a BH with isotropic energy emission of the order of $E_{\text {iso }} \gtrsim 10^{52} \mathrm{erg}$. Gravitational waves are produced (Oliveira et al. 2014) together with $\mathrm{GeV}$ emission from the accretion onto the Kerr BH (Ruffini et al. 2018). It has been shown (Becerra et al. 2018) that the accretion onto the NS generates neutrino-antineutrino emission in the case of long GRBs, and this emission has been explained as being due to $e^{-} e^{+}$-pair annihilation.

In this work, we apply the Fireshell Model to explain neutrino emission in S-GRBs. We describe the conditions under which the neutrino emission takes place, and we analyze the effects of flavor oscillations and decoherence on neutrinos on their way from the source to the observer on Earth.

The work is organized as follows: In Section 2, we describe the model. In Section 3, we derive the expressions for the electron and neutrino number densities, following a statistical treatment. In Section 4, we compute the electron and neutrino fluxes at the source. In Section 5, we analyze the effects of neutrino-flavor oscillations in vacuum, from the moment in which neutrinos are produced up to the time they reach the external crust. In Section 6, we analyze the effects of neutrinoflavor oscillations in matter, as they propagate through the crust and interact with baryons. In Section 7, we introduce the mechanism of decoherence, as neutrinos that leave the crust and propagate through the universe toward the observer interact with background intergalactic particles. We calculate the detected flux on Earth and compare it with the flux at the source. The results are presented and discussed in Section 7.3. Finally, in Section 8 we draw our conclusions.

\section{The Model}

A typical scenario for S-GRBs within the Fireshell Model is depicted in Figure 1. Two NSs of masses $M_{1}$ and $M_{2}$, typically of the order of 1.6-2 $M_{\odot}$, start spiraling together until they merge giving birth to a $\mathrm{BH}$ due to gravitational collapse. Let us consider for simplicity $M_{1}=M_{2}$. Only the core collapses, leaving a thin crust of a fraction of a solar mass. In the vacuum between the crust and the $\mathrm{BH}$ event horizon, a strong electric field is generated due to charge separation. When this field reaches the critical value, $E_{c}$, vacuum polarization takes place generating an $e^{-} e^{+}$-plasma. Typical densities for the electronpositron plasma are of the order of $10^{33}$ particles $\mathrm{cm}^{-3}$. Some of these pairs annihilate, giving neutrinos and antineutrinos that propagate outward, first in vacuum then through the crust 


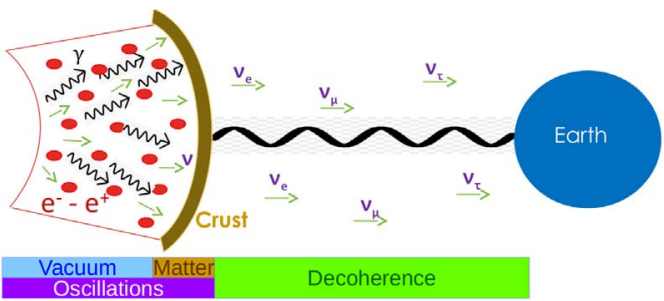

Figure 1. Schematic representation (not to scale) of neutrino emission in the Fireshell Model. The two merging NSs evolve into a BH. Vacuum polarization generates $e^{-} e^{+}$-pairs that annihilate to photons and neutrinos in the region delimited by the crust. Neutrinos travel toward the observer, oscillate in flavor, and interact with intergalactic neutrinos.

formed by $e^{-}$, protons, and neutrons and finally through the intergalactic medium until they reach the observer on Earth (SNO Collaboration 2000; Halzen \& Klein 2010). Another fraction of the $e^{-} e^{+}$-pairs produce thermal photons. Since at this stage the system is still opaque to radiation, the radiation pressure increases making the plasma expand until it reaches the crust. The whole system continues to expand until it reaches transparency. At this point the thermal photons escape. This is seen as a thermal spike in the spectrum called proper GRB (P-GRB; Ruffini et al. 2001). The remaining material continues to expand while interacting with the circumburst medium producing the prompt emission.

Table 1 shows the values of the parameters of our model.

\section{Neutrino Number Density and Energy}

In order to calculate the number density and energy of the neutrinos created during the merging of the two NSs, we follow a statistical treatment. We treat the neutrinos as a Fermi-Dirac gas in thermodynamical equilibrium at temperature $k T=$ $2 \mathrm{MeV}$ (Ruffini et al. 1999). The neutrino emission zone is the same as the one occupied by the $e^{-} e^{+}$-plasma, a shell that extends from the $\mathrm{BH}$ event horizon $\left(r_{\mathrm{BH}} \approx 10^{5} \mathrm{~cm}\right)$ to the crust $\left(r_{\text {crust }} \approx 10^{10} \mathrm{~cm}\right)$.

The Fermi-Dirac distribution function for $T \neq 0$ is given by

$$
f(E)=\frac{1}{e^{(E-\mu) / k_{\mathrm{B}} T}+1},
$$

where $k_{\mathrm{B}}$ is the Boltzmann constant and $E$ is the fermion energy. The parameter $\mu$ is known as the Fermi level. In the limit $T \rightarrow 0, f(E)$ becomes a step function $\theta(E-\mu)$ : all the energy levels with $E<\mu$ are occupied, while all the others are empty.

\subsection{Electrons}

In the relativistic case, the energy of the electrons is $E=\sqrt{p^{2} c^{2}+m^{2} c^{4}}$, the momentum in terms of the energy is given by $p=\frac{1}{c} \sqrt{E^{2}-m^{2} c^{4}}$, and $d p=\frac{1}{c} \frac{E d E}{\sqrt{E^{2}-m^{2} c^{4}}}$. The number of particles in the system is given by the expression

$$
N=\int f(E) d \Omega=\frac{4 \pi V g_{s}}{(2 \pi \hbar)^{3}} \int_{0}^{\infty} \frac{p^{2} d p}{e^{\left(E-\mu_{e}\right) / k_{\mathrm{B}} T}+1} .
$$

Here,

$$
d \Omega=\frac{d \boldsymbol{q} d \boldsymbol{p}}{(2 \pi \hbar)^{3}}=\frac{4 \pi V}{(2 \pi \hbar)^{3}} p^{2} d p
$$

Table 1

Parameters of Our Model

\begin{tabular}{lcc}
\hline \hline Parameter & Symbol & Value \\
\hline NS radius $[\mathrm{km}]$ & $R_{\mathrm{NS}}$ & 10 \\
NS mass $\left[M_{\odot}\right]$ & $M_{\mathrm{NS}}$ & 2 \\
$e^{ \pm}$plasma density [part cm $\left.{ }^{-3}\right]$ & $N_{e}^{ \pm}$ & $10^{33}$ \\
$e^{ \pm}$plasma temperature $[\mathrm{MeV}]$ & $\mathrm{kT}$ & 2.0 \\
$\mathrm{BH}$ radius [cm] & $r_{\mathrm{BH}}$ & $3.3 \times 10^{5}$ \\
Crust internal radius [cm] & $r_{\text {int }}$ & $1.69 \times 10^{8}$ \\
Crust external radius [cm] & $r_{\text {ext }}$ & $1.2 \times 10^{10}$ \\
Source-detector distance $[\mathrm{cm}]$ & $D_{L}$ & $10^{28}$ \\
Mass of the crust $\left[M_{\odot}\right]$ & $M_{\text {crust }}$ & 0.1 \\
Density of the crust $\left[\mathrm{g} \mathrm{cm}^{-3}\right]$ & $\rho_{\text {crust }}$ & 27.47 \\
Proton density in the crust $\left[\mathrm{part} \mathrm{cm} \mathrm{cm}^{-3}\right]$ & $N_{p}$ & $0.25 \rho_{\text {crust }}$ \\
Neutron density in the crust $\left[\mathrm{part} \mathrm{cm}^{-3}\right]$ & $N_{n}$ & $0.25 \rho_{\text {crust }}$ \\
$e^{-}$density in the crust $\left[\mathrm{part} \mathrm{cm}^{-3}\right]$ & $N_{e}$ & $0.50 \rho_{\text {crust }}$ \\
\hline
\end{tabular}

is the volume element in phase space and $g_{s}=2$ is the spin degeneracy factor. Changing variables to $x=E-m c^{2}$ yields the number density $\rho_{e}=N / V$ :

$$
\rho_{e}=\frac{(2 m)^{3 / 2}}{2 \pi^{2} \hbar^{3}} \int_{0}^{\infty} \frac{x^{1 / 2}\left(1+x / 2 m c^{2}\right)^{1 / 2}\left(1+x / m c^{2}\right) d x}{1+e^{\left(x+m c^{2}-\mu_{e}\right) / k T}} .
$$

By making the substitutions $\eta_{e}=\left(\mu_{e}-m c^{2}\right) / k T, w=x / k T$, and $\beta=k T / m c^{2}$, Equation (3) becomes

$$
\rho_{e}=\frac{(2 m k T)^{3 / 2}}{2 \pi^{2} \hbar^{3}}\left[F_{1 / 2}\left(\eta_{e}\right)+\beta F_{3 / 2}\left(\eta_{e}\right)\right],
$$

where

$$
F_{r}=\int_{0}^{\infty} \frac{x^{r}(1+x \beta / 2)^{1 / 2} d x}{1+e^{x-\eta_{e}}}
$$

for $r=1 / 2,3 / 2,5 / 2$, etc.

A similar expression is obtained for the mean energy of the electrons as a function of temperature and density:

$$
\frac{\left\langle E_{e}\right\rangle}{\rho_{e}}=m c^{2} \frac{F_{1 / 2}+2 \beta F_{3 / 2}+\beta^{2} F_{5 / 2}}{F_{1 / 2}+\beta F_{3 / 2}} .
$$

\subsection{Neutrinos}

Neutrinos have negligible masses compared to their energy $\left(E \gg m c^{2}\right)$, so $E \approx p c$. Following the same procedure as in Section 3.1 we find for the neutrino number density

$$
\rho_{\nu}=\frac{N_{\nu}}{V}=\frac{4 \pi g_{s}\left(k_{\mathrm{B}} T\right)^{3}}{(2 \pi \hbar c)^{3}} F_{2}\left(\eta_{\nu}\right),
$$

where

$$
F_{n}=\int_{0}^{\infty} \frac{x^{n} d x}{1+e^{x-\eta_{\nu}}}
$$

for $n=1,2,3, \ldots$ and $\eta_{\nu}=\mu_{\nu} / k T$.

The neutrino mean energy is given by

$$
\left\langle E_{\nu}\right\rangle=\frac{\left(k_{\mathrm{B}} T\right)^{4}}{\pi^{2}(\hbar c)^{3}} F_{3}\left(\eta_{\nu}\right) ;
$$


$f(E)$ vs $E$

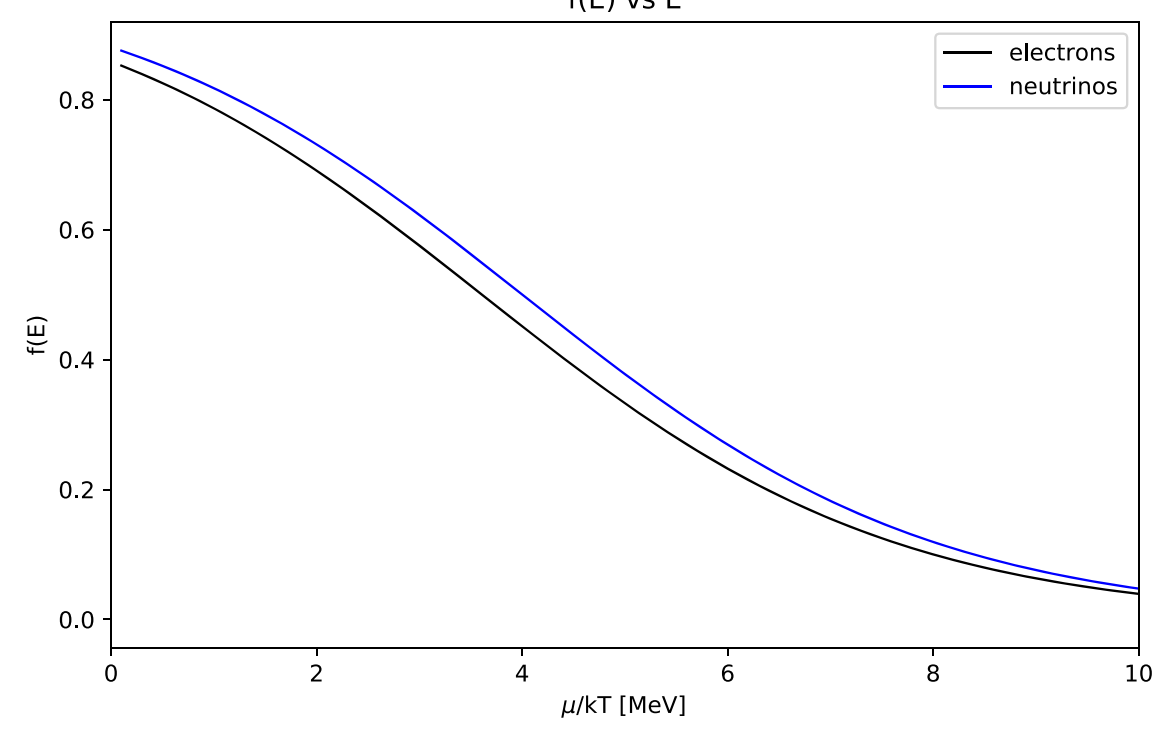

Figure 2. Neutrino (upper curve) and electron (lower curve) occupation numbers for $k T=2 \mathrm{MeV}$ (see Equation (1)).
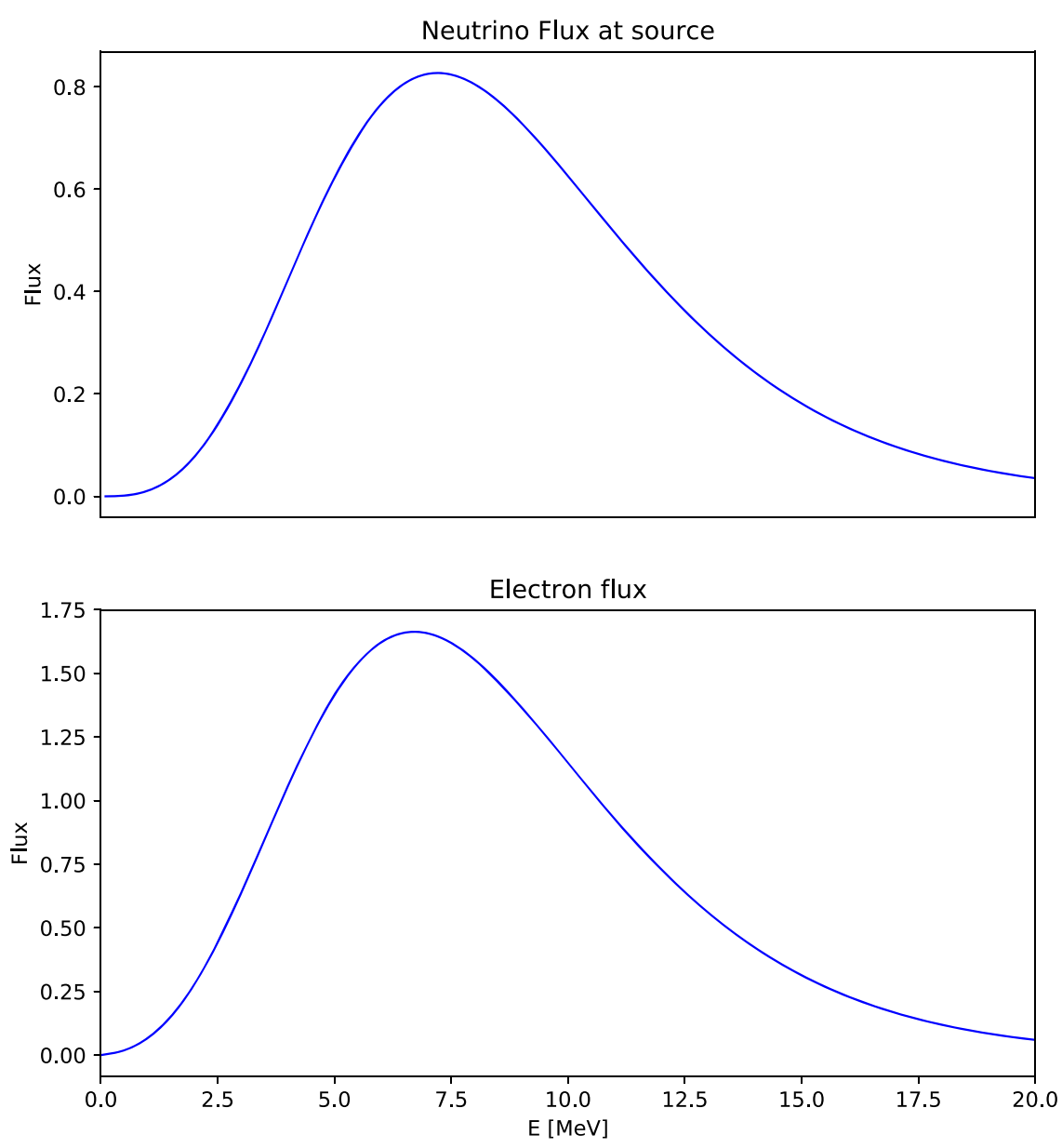

Figure 3. Electron and neutrino fluxes, as a function of the energy, at the source (see Equation (9)).

thus, the mean energy per neutrino is given by

$$
\frac{\left\langle E_{\nu}\right\rangle}{\rho_{\nu}}=k T\left(\frac{F_{3}\left(\eta_{\nu}\right)}{F_{2}\left(\eta_{\nu}\right)}\right)
$$

\section{Electron and Neutrino Fluxes at Source}

With the parameters given in Table 1 and the formalism presented in Section 3 we have performed a numerical search to determine the electron and neutrino chemical potentials $\mu_{e}$ and $\mu_{\nu}$, and with them the mean energies and spectral functions (Cox \& Giuli 1968). 
The numerical search gives from Equations (3) and (6) the best values of $\eta_{e}$ and $\eta_{\nu}$ for a given density. The results are $\eta_{e}=1.75$ and $\eta_{\nu}=2.0$, corresponding to $\mu_{e}=4.01 \mathrm{MeV}$ and $\mu_{\nu}=4.00 \mathrm{MeV}$. Figure 2 shows the occupation numbers $f(E)$ of Equation (1) for a plasma temperature of $2 \mathrm{MeV}$ (Ruffini et al. 1999).

We have calculated the electron and neutrino fluxes inside the $e^{-} e^{+}$-plasma. Each flux is given by the ratio

$$
F_{e, \nu}=\frac{1}{\rho_{e, \nu}} \frac{d\left\langle E_{e, \nu}\right\rangle}{d E} .
$$

Figure 3 shows the results of Equation (9) for electron and neutrino fluxes in the region of the $e^{-} e^{+}$-plasma.

\section{Neutrino Oscillations in Vacuum}

As soon as they are created, neutrinos start to propagate outward at nearly the speed of light from the region close to the event horizon toward the crust. This region is opaque to radiation, but nothing prevents neutrinos from escaping. Because of the geometry of the source (see Figure 1) we shall consider propagation and oscillations in vacuum in the inner region between the $\mathrm{BH}$ and the crust.

Neutrinos oscillate because the flavor states in which they are created are a superposition of mass eigenstates. Because they have different masses they evolve with different phases.

The Hamiltonian in the mass basis is given by

$$
H_{m}=\left[\begin{array}{lll}
m_{1} & 0 & 0 \\
0 & m_{2} & 0 \\
0 & 0 & m_{3}
\end{array}\right] .
$$

The mass hierarchies are denoted, as usual: $m_{1} \leqslant m_{2} \ll m_{3}$ (normal hierarchy), $m_{1} \ll m_{2} \leqslant m_{3}$ (inverted hHierarchy), or $m_{1} \approx m_{2} \approx m_{3}$ (degenerate hierarchy). Adopting the normal hierarchy and setting $m_{1}=0$ yields $m_{2}=0.00858 \mathrm{eV}$ and $m_{3}=0.0506 \mathrm{eV}$.

The neutrino mass Hamiltonian $H_{m}=\operatorname{diag}\left(m_{1}, m_{2}, m_{3}\right)$ is transformed to the flavor basis by applying upon it the mixing matrix (Bilenky 2000; Kersten \& Smirnov 2016)

$$
\begin{aligned}
& U \\
& =\left[\begin{array}{lcc}
c_{12} c_{13} & s_{12} c_{13} & s_{13} e^{-\delta} \\
-s_{12} c_{23}-c_{12} s_{23} s_{13} e^{\delta} & c_{12} c_{23}-s_{12} s_{23} s_{13} e^{\delta} & s_{23} c_{13} \\
s_{12} s_{23}-c_{12} c_{23} s_{13} e^{\delta} & -c_{12} s_{23}-s_{12} c_{23} s_{13} e^{\delta} & c_{23} c_{13}
\end{array}\right],
\end{aligned}
$$

where $c_{i j}\left(s_{i j}\right)$ is the cosine (sine) of the mixing angles $\theta_{i j}$. Therefore,

$$
H_{f}=U H_{m} U^{-1}
$$

The parameters entering $U$ and $H_{m}$ are listed in Table 2. In the present analysis we have taken, for the Dirac CP-violating phase, the value $\delta / \pi=0$. Furthermore, and in order to separate the electronic flavor from linear combinations of the $\mu$ and $\tau$ ones, we apply to the flavor Hamiltonian the decoupling matrix
Table 2

Neutrino-Oscillation Parameters, for the Normal (NH) and Inverted (IH) Mass Hierarchies

$\sin ^{2}\left(\theta_{12}\right)=0.297$

$\sin ^{2}\left(\theta_{13}\right)=0.0215$

$\sin ^{2}\left(\theta_{23}\right)=0.425(\mathrm{NH})$

$\sin ^{2}\left(\theta_{23}\right)=0.589(\mathrm{IH})$

$\delta_{\mathrm{atm}}^{2}=m_{3}^{2}-m_{1}^{2}=2.56 \times 10^{-3} \mathrm{eV}^{2}$

$\delta_{\text {solar }}^{2}=m_{2}^{2}-m_{1}^{2}=7.37 \times 10^{-5} \mathrm{eV}^{2}$

Note. Solar $\left(\delta_{\text {solar }}^{2}\right)$ and atmospheric $\left(\delta_{\text {atm }}^{2}\right)$ squared mass differences and mixing angles $\left(\theta_{i j}\right)$ are listed in the table. The values are taken from http://pdg.lbl. gov/2017/reviews/rpp2017-rev-neutrino-mixing.pdf.

(Kersten \& Smirnov 2016)

$$
D=\left[\begin{array}{ccc}
1 & 0 & 0 \\
0 & 1 / \sqrt{2} & 1 / \sqrt{2} \\
0 & -1 / \sqrt{2} & 1 / \sqrt{2}
\end{array}\right] .
$$

The "decoupled" flavor Hamiltonian reads

$$
H_{f}^{d e c}=D H_{f} D^{-1},
$$

and from the diagonalization of this Hamiltonian we obtain the eigenvalues and eigenvectors for electron-neutrinos $\nu_{e}$ and nonelectronic neutrinos $\nu_{x}=\frac{1}{\sqrt{2}}\left(\nu_{\mu} \pm \nu_{\tau}\right)$.

\section{Oscillations in Matter}

Following the model sketch in Figure 1, neutrinos oscillate in vacuum until they reach the internal radius of the crust. At this point, they interact with matter. We assume the crust is formed by electrons, protons, and neutrons in the amounts given in Table 1. A matter Hamiltonian must be added to the flavor Hamiltonian in vacuum. For the matter Hamiltonian we consider a diagonal one:

$$
H_{\text {mat }}=\operatorname{diag}\left(V_{m}, 0,0\right),
$$

where $V_{m}=\sqrt{2} G_{\mathrm{F}}\left(N_{e}+N_{p}+N_{n}\right)$ is the matter potential, $G_{\mathrm{F}}=8.963 \times 10^{-44} \mathrm{MeV} \mathrm{cm}^{3}$ is the Fermi constant, and $N_{e}$, $N_{p}$ and $N_{n}$ are the electron, proton, and neutron densities in the crust, respectively.

Because of the thickness of the crust $\left(\approx 10^{2} \mathrm{~cm}\right)$ the interactions with matter are negligible despite the values of the baryon densities. Therefore, we shall not take these interaction into account in our analysis.

\section{Decoherence}

Once the neutrinos arrive at the external radius of the crust they continue their way to the detector on Earth. As the distance that they have to travel is of the order of $D_{L} \approx 10^{28} \mathrm{~cm}$, corresponding to typical S-GRB redshifts (Ruffini et al. 2016), decoherence effects (Schlosshauer 2007) may take place due to interactions of the source neutrinos with neutrinos in the cosmic background. Decoherence effects are relevant in the reconstruction of the sequence of events starting from the primordial production of neutrinos and ending at their detection. What we would like to evaluate quantitatively is the difference between the composition of neutrinos of the source, as dictated by the neutrino-oscillation mechanism, and their 
time evolution governed by decoherence. The decoherence mechanism we have in mind in not kinematic and, as we said before, it is due to interactions with other particles like neutrinos that fill the space between the source and the detector. In order to achieve this goal we shall proceed to:

1. calculate the density matrix from the diagonalization of the flavor Hamiltonian (Equation (14));

2. construct the time evolution matrix that determines the time dependence of the density matrix; and

3. calculate the probability of detecting neutrinos of a given flavor on Earth.

In what follows, we present the corresponding theoretical details.

\subsection{Flavor Eigenstates at $t=0$}

The density matrix for electron-neutrinos leaving the crust is

$$
\rho_{\nu_{e}}=\left|\phi_{\nu_{e}}\right\rangle \otimes\left\langle\left.\phi_{\nu_{e}}\right|_{(t=0)}\right. \text {. }
$$

With the amplitudes of the electron-neutrino eigenvalue obtained by the diagonalization of $H_{f}$, Equation (16) is readily calculated.

The density matrix (Equation (16)) is that of a pure state, that is, $\rho^{2}=\rho$, and its diagonalization yields the survival probabilities of the electron-, muon-, and tau-neutrino channels, respectively.

\subsection{Time Dependence of the Density Matrix}

To calculate the time dependence of the density matrix for neutrinos leaving the crust we add to the flavor Hamiltonian the interaction of the electron-neutrinos with the environment. For this, we follow the formalism presented in Bes \& Civitarese (2017) and Schlosshauer (2007). Accordingly, we construct the matrix

$$
A=H_{f}^{\mathrm{dec}}+\operatorname{diag}\left(B \lambda_{\text {coup }} / 2,0,-B \lambda_{\text {coup }} / 2\right),
$$

where $\lambda_{\text {coup }}$ is the coupling constant and $B$ is a constant field acting on the neutrinos. The diagonalization of $A$ leads to the eigenvalues and eigenvectors needed to construct the evolution matrix $U(t)$ (Schlosshauer 2007), which is defined by the expression

$$
U(t)=V \operatorname{diag}\left(e^{i \Omega_{n} t}\right) V^{-1},
$$

where $V$ is the matrix of eigenvectors of $A, \Omega_{n}, n=1,2,3$, the associated eigenvalues, being both $V$ and $\Omega_{n}$ functions of the strength $B$. In writing Equation (18), we use $\hbar=1$. In this picture the density matrix $\rho_{\nu_{e}}$ of Equation (16) evolves with time as

$$
\rho(t, B)=U(t) \rho_{\nu_{e}} U^{-1}(t) .
$$

If the strength $B$ is distributed like a Gaussian around $B=0$ with standard deviation $\sigma$, we integrate the matrix $\rho(t, B)$ in B so that its elements $[\rho(t)]_{i j}$ are

$$
[\rho(t)]_{i j}=\int[\rho(t, B)]_{i j} \frac{e^{-B^{2} / 2 \sigma^{2}}}{\sqrt{2 \pi} \sigma} d B .
$$

A last diagonalization of $\rho(t)$ for $t$ is sufficiently large, of the order of $L / c$ being $L$ the distance from the source to the detector and $c$ the speed of light, $c$, and leads to the survival probabilities of neutrinos of a given flavor, in this case, of electron-neutrinos. These probabilities are needed in order to renormalize the neutrino flux at the Earth, as explained below.

$$
\text { 7.3. Results for } \rho(t=0) \text { and } \rho(t)
$$

For the normal hierarchy and masses $m_{1}=0, m_{2}=$ $0.00866 \mathrm{eV}, m_{3}=0.0495 \mathrm{eV}$, and $\delta=0$ in Equation (11), we get

$$
\rho_{\nu_{e}}(t=0)=\left[\begin{array}{ccc}
0.680 & -0.124 & 0.449 \\
-0.124 & 0.022 & -0.082 \\
0.449 & -0.082 & 0.297
\end{array}\right] .
$$

The diagonalization of $\rho(t=0)$ gives

$$
P_{\nu_{e} \rightarrow \nu_{e}}(t=0)=1, P_{\nu_{e} \rightarrow \nu_{x}}(t=0)=0 .
$$

To illustrate the effect of decoherence we calculate the time evolution given by Equation (20) with $\lambda_{\text {coup }}=1.0$ and $\sigma=20$.

For a sufficiently large number of oscillations in the presence of the interactions due to the background and for the chosen parameterization, the density matrix is given by

$$
\begin{aligned}
\rho(t) & =\rho_{(R e)}(t)+i \rho_{(I m)}(t) \\
& =\left[\begin{array}{ccc}
0.680 & -0.0005 & 0.00002 \\
-0.0005 & 0.022 & -0.0002 \\
0.00002 & -0.0002 & 0.296
\end{array}\right] \\
& +i\left[\begin{array}{ccc}
0 & 0.005 & -0.0008 \\
-0.005 & 0 & -0.0003 \\
0.0008 & 0.0003 & 0
\end{array}\right],
\end{aligned}
$$

which is no longer the density matrix of a pure state, as $\rho^{2} \neq \rho$. Diagonalization of this matrix gives the survival probabilities

$$
\begin{aligned}
& P_{\nu_{e} \rightarrow \nu_{e}}(t \rightarrow \infty)=0.67871, \\
& P_{\nu_{e} \rightarrow \nu_{x}}(t \rightarrow \infty)=0.32129 .
\end{aligned}
$$

Figure 4 shows the elements of the density matrix as a function of time, as the system evolves to the pointer states. The fact that the matrix $\rho(t)$ loses its pure-state nature due to decoherence is better illustrated by the results shown in Figure 5, where the final pointer states are identified by means of the probabilities $P_{\nu_{e} \rightarrow \nu_{e}}$ and $P_{\nu_{e} \rightarrow \nu_{x}}$.

The neutrino flux, for neutrinos emitted at the source (see Figure 3), should then be renormalized to account for the evolution of $\rho$ from pure to pointer states. This is done by multiplying the curves of Figure 3 by the probabilities (24) and (25). The results are shown in Figure 6.

We shall calculate the characteristic wavelengths of flavor oscillations to compare them with the size of the regions where the effects take place.

In the case of flavor oscillations, the amplitude of the electron flavor survival $A_{\nu_{e} \rightarrow \nu_{e}}$ is given by

$$
\operatorname{Re}\left(A_{\nu_{e} \rightarrow \nu_{e}}\right)=\left|U_{\nu 1}\right|^{2}+\sum_{j=2,3}\left|U_{\nu j}\right|^{2} \cos \left(\Delta_{1 j}\right)
$$

and

$$
\operatorname{Im}\left(A_{\nu_{e} \rightarrow \nu_{e}}\right)=\sum_{j=2,3}\left|U_{\nu j}\right|^{2} \sin \left(\Delta_{1 j}\right)
$$



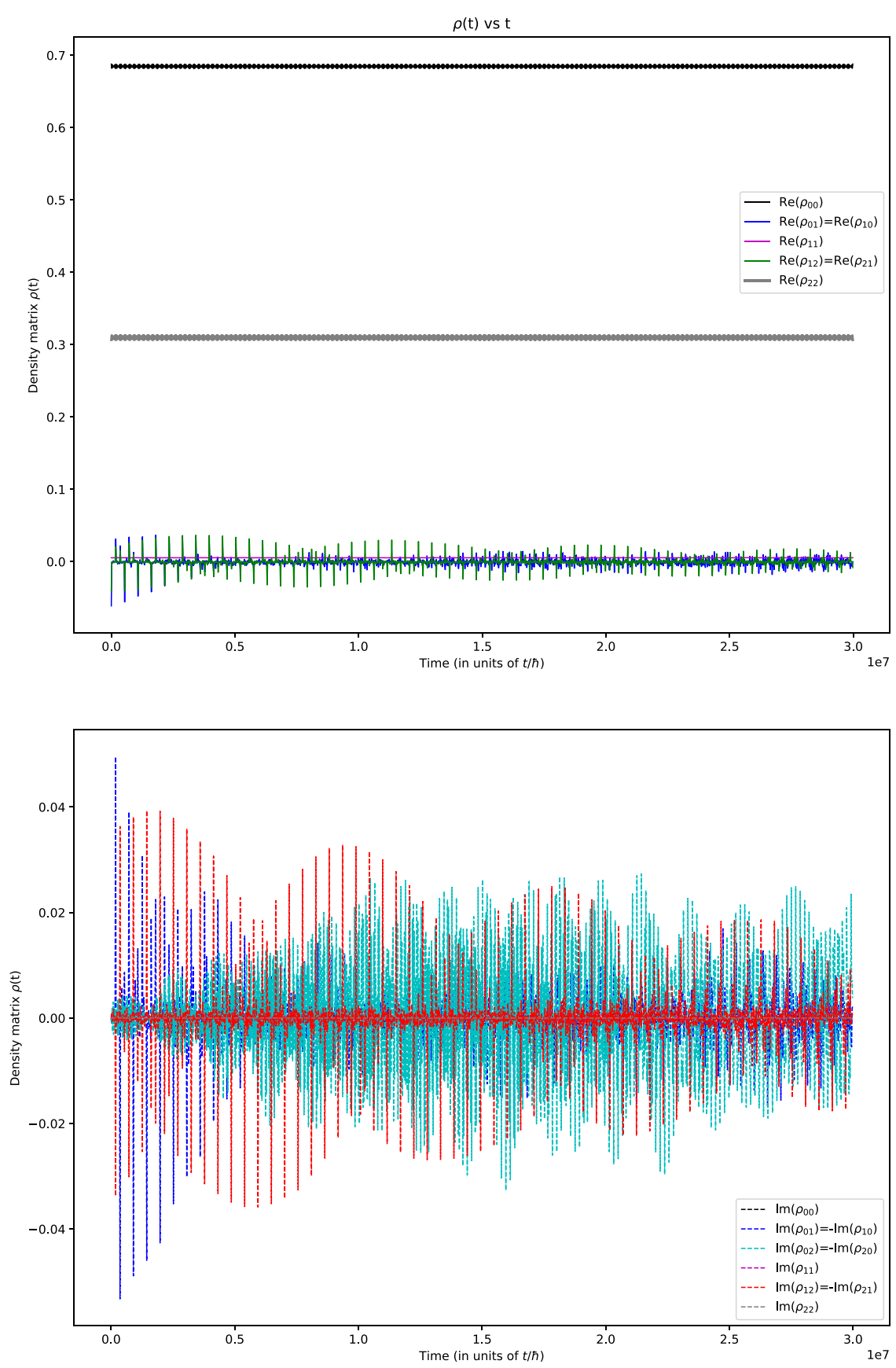

Figure 4. Real (top panel) and imaginary (bottom panel) matrix elements (Equation (20)) of the density matrix as a function of time for the three-flavor scheme. We used the following parameters: $\sigma=20$ and $\lambda_{\text {coup }}=1.0$ for the Gaussian function and the coupling to the environment.

for the real and imaginary parts of the amplitude, respectively, with

$$
\Delta_{1 j}=\frac{\left(m_{j}^{2}-m_{1}^{2}\right) c^{4} t}{2 E \hbar}=\frac{\delta_{1 j}^{2} t}{2 E \hbar}
$$

To make a rough estimation of the period of oscillations, we take the squared mass difference $\delta_{12}^{2}$ in the normal hierarchy and write for the period $T_{\text {osc }}$ (Kersten \& Smirnov 2016)

$$
T_{\mathrm{osc}}=\frac{8 \pi E \hbar c}{\delta_{12}^{2} c^{4}} .
$$

The corresponding wavelength for flavor oscillations will then be

$$
\lambda_{\mathrm{osc}}=c T_{\mathrm{osc}}
$$

For the neutrino mean energy obtained in our calculations, $\langle E\rangle=3.98 \mathrm{MeV}$, we have $\lambda_{\text {osc }}=1.98 \times 10^{8} \mathrm{~cm}$. This is much larger than the distance from the event horizon to the external part of the crust $\left(\approx 10^{5} \mathrm{~cm}\right)$, therefore confirming the pure-state nature of the density matrix (Equation (16)) for neutrinos leaving the source. 


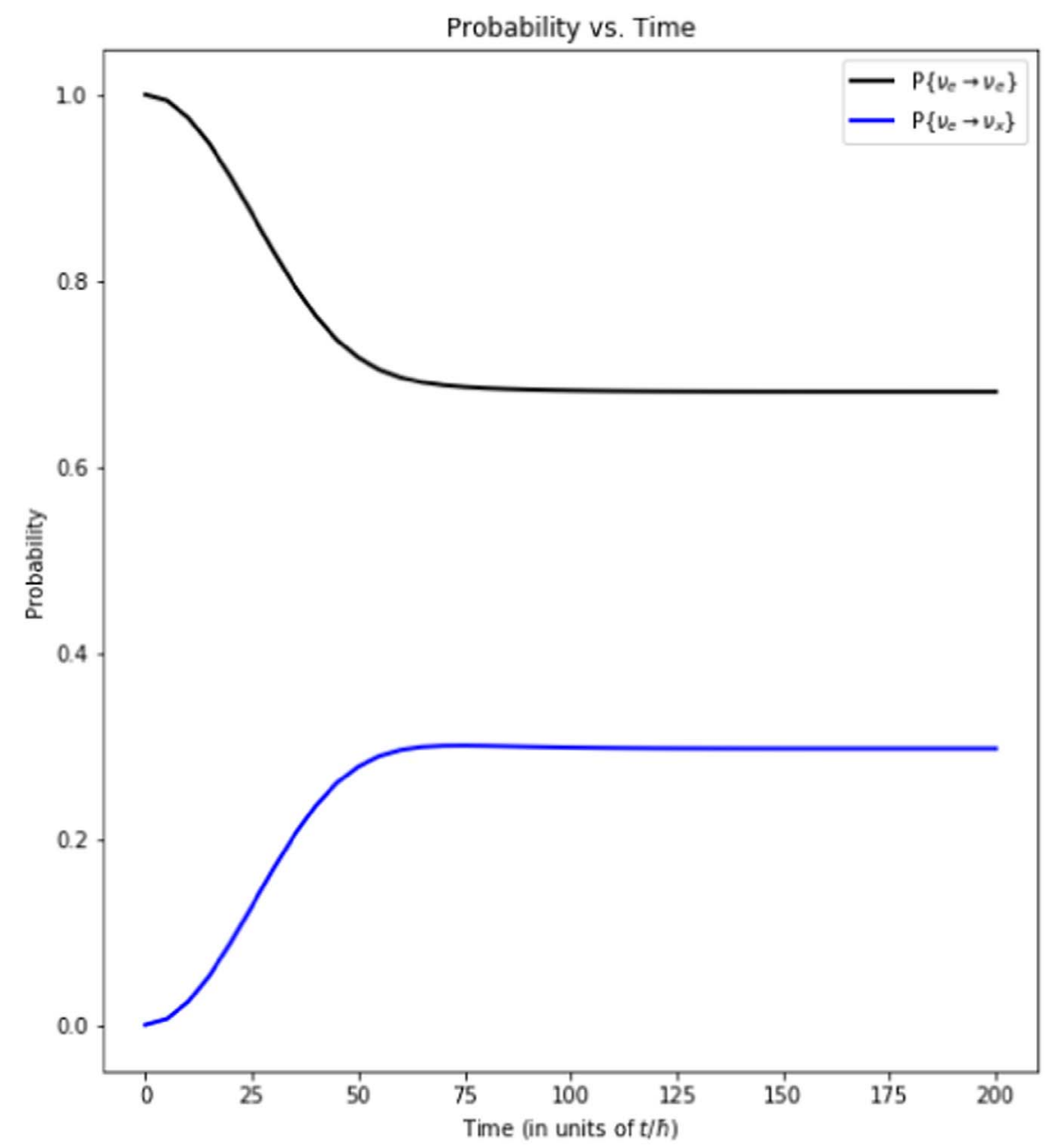

Figure 5. Eigenvalues of the density matrix $\rho(t)$. From the initial values $P_{\nu_{e} \rightarrow \nu_{e}}(t=0)=1$ and $P_{\nu_{e} \rightarrow \nu_{e}}(t=0)=0$, the probabilities evolve to the asymptotic values $P_{\nu_{e} \rightarrow \nu_{e}}=0.67871$ and $P_{\nu_{e} \rightarrow \nu_{x}}=0.32129$ of the pointer states.

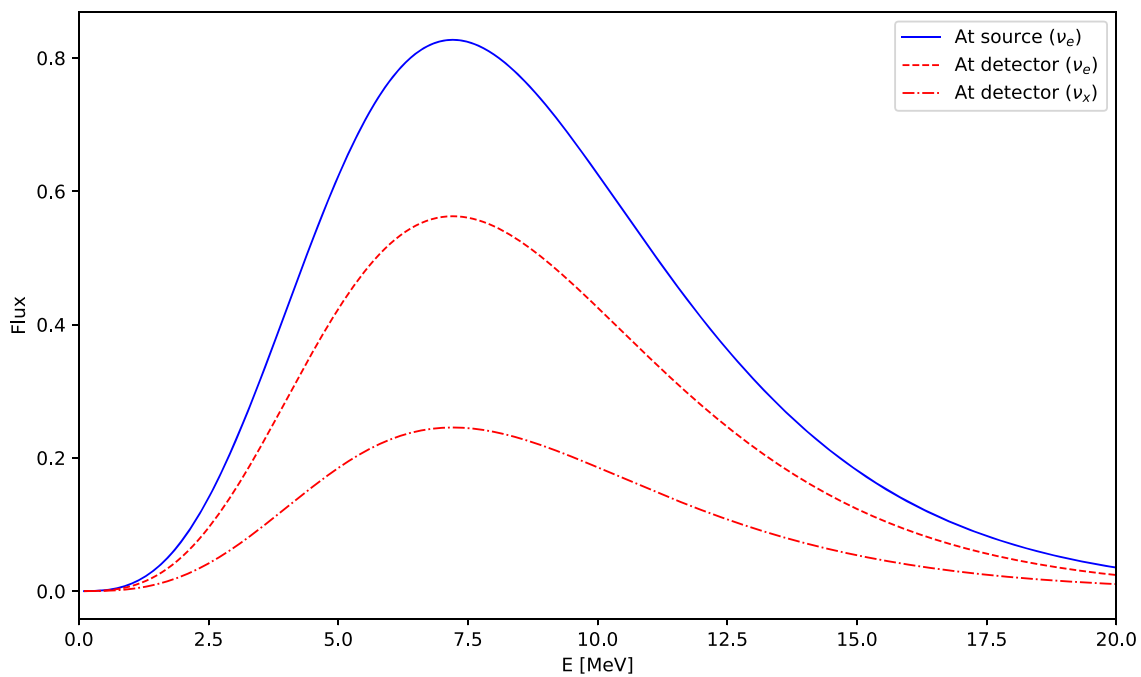

Figure 6. Neutrino flux at the moment of the creation at source (blue; only electron-neutrinos are created according to our model) and at the detector on Earth (red; due to decoherence effects, some electron-neutrinos disappear and $x$ neutrinos are created).

\subsection{About the Observability of the Emitted Neutrinos}

The results that we have presented so far show that the survival probabilities for the emitted electron-neutrinos change considerably, as do the calculated fluxes at the source and at the detector. As mentioned before, Equation (9) gives the number of particles (electrons or neutrinos) with energies in the interval $E \pm \Delta E$.

Köpke \& IceCube Collaboration (2011) performed a simulation of a supernova (SN) event at a distance of $10 \mathrm{kpc}$ with a total emitted energy of $2.9 \times 10^{53} \mathrm{erg}$, starting from a 
$20 M_{\odot}$ progenitor and considering inverse beta decay, neutron capture, and positron annihilation as the main channels for neutrino interaction. They obtain a mean energy of the order of $15 \mathrm{MeV}$ and a rate of $\approx 1.7 \times 10^{5}$ counts s $^{-1}$. In our case, we consider an NS-NS merger leading to a $2.7 M_{\odot}$ progenitor at a redshift $z=0.9$, which corresponds to a distance of $\sim 3800 \mathrm{Mpc}$ (or $10^{28} \mathrm{~cm}$, as stated in Table 1), just like GRB 090510 (Rau et al. 2009). The only channel considered for neutrino production in our model is $e^{-}-e^{+}$annihilation (we intend to extend the model by considering more production channels that contribute to the total neutrino flux in a future work). We obtain a neutrino mean energy per particle of $3.98 \mathrm{MeV}$. The rate at the detector is thus of the order of $10^{-4}$ events $\mathrm{s}^{-1}$, which is far from being detected with the current IceCube sensitivity and effective area. However, this may be achieved by future detector generations. What we would like to emphasize is that our calculations give us a mean neutrino energy that falls in the range of SN neutrinos (see Figure 1 of Spiering 2012).

\section{Conclusions}

In this work, we have investigated the processes leading to the emission of neutrinos in short-GRB progenitors. Following the discussions advanced in the literature (Bianco \& Ruffini 2008) we have modeled the system so that the $e^{-} e^{+}$-plasma is the main source of neutrinos. These neutrinos travel through the region between the $\mathrm{BH}$ event horizon and the crust, their density matrix being that of pure states described by neutrino flavor-oscillations. Because of the astronomical scale of the distance between the source and the detector on Earth, decoherence effects due to interaction with the cosmic background may become important. We have calculated these effects by adopting a Gaussian model to incorporate the cosmic background.

The present calculations give a mean neutrino energy that falls in the range of SN neutrinos. The value of the predicted neutrino flux is still far away from observation, but considering the continuous advances in detector technology, it could be reachable by future generations of experiments.

Further work is in progress concerning the time delay between neutrino and photon emission in GRBs.

The authors would like to thank Dr. A. Marinelli for useful discussions. This work has been partially supported by the
National Research Council of Argentina (CONICET) by the grant PIP 616, and by the Agencia Nacional de Promoción Científica y Tecnológica (ANPCYT) PICT 140492. A.V.P and O.C. are members of the Scientific Research career of the CONICET.

\section{ORCID iDs}

A. V. Penacchioni (i) https://orcid.org/0000-0001-7816-3668

\section{References}

Becerra, L., Guzzo, M. M., Rossi-Torres, F., et al. 2018, ApJ, 852, 120 Berger, E. 2014, ARA\&A, 52, 43

Bes, D. R., \& Civitarese, O. 2017, in AIP Conf. Ser. 1849, Workshop on Calculation of Double-Beta-Decay Matrix Elements (Medex'17), ed. O. Civitarese et al. (Melville, NY: AIP), 020006

Bianco, C. L., Bernardini, M. G., Caito, L., et al. 2008a, in AIP Conf. Ser. 966 Relativistic Astrophysics, ed. C. L. Bianco \& S.-S. Xue (Melville, NY: AIP), 12

Bianco, C. L., Bernardini, M. G., Caito, L., et al. 2008b, in AIP Conf. Ser. 1065, 2008 Nanjing Gamma-Ray Burst Conference, ed. Y.-F. Huang, Z.-G. Dai, \& B. Zhang (Meville, NY: AIP), 223

Bianco, C. L., \& Ruffini, R. 2008, in The Eleventh Marcel Grossmann Meeting On Recent Developments in Theoretical and Experimental General Relativity, Gravitation and Relativistic Field Theories, ed. H. Kleinert, R. T. Jantzen, \& R. Ruffini (Singapore: World Scientific), 1989

Bilenky, S. M. 2000, arXiv:hep-ph/0001311

Cox, J. P., \& Giuli, R. T. 1968, Principles of stellar structure (New York: Gordon and Breach)

Enderli, M., Bianco, C. L., Izzo, L., et al. 2014, in Proc. of Swift: 10 Years of Discovery (SWIFT 10), ed. P Caraveo et al. (Trieste: SISSA), 73

Halzen, F., \& Klein, S. R. 2010, RScI, 81, 081101

Kersten, J., \& Smirnov, A. Y. 2016, EPJC, 76, 339

Köpke, L. \& IceCube Collaboration 2011, JPhCS, 309, 012029

Narayan, R., Paczynski, B., \& Piran, T. 1992, ApJL, 395, L83

Oliveira, F. G., Rueda, J. A., \& Ruffini, R. 2014, ApJ, 787, 150

Piran, T. 1999, PhR, 314, 575

Rau, A., McBreen, S., \& Kruehler, T. 2009, GCN, 9353

Ruffini, R., Bianco, C. L., Fraschetti, F., Xue, S.-S., \& Chardonnet, P. 2001, ApJL, 555, L117

Ruffini, R., Salmonson, J. D., Wilson, J. R., \& Xue, S.-S. 1999, A\&A, 350, 334

Ruffini, R., Muccino, M., Aimuratov, Y., et al. 2016, ApJ, 831, 178

Ruffini, R., Muccino, M., Aimuratov, Y., et al. 2018, arXiv:1802.07552

Schlosshauer, M. 2007, Decoherence and the Quantum-to-Classical Transition (Berlin: Springer)

SNO Collaboration 2000, in AIP Conf. Ser. 533, Next Generation Nucleon Decay and Neutrino Detector: Nnn99, ed. M. V. Diwan \& C. K. Jung (Melville, NY: AIP), 118

Spiering, C. 2012, EPJH, 37, 515 